\title{
Covering a nontaming knot by the unlink
}

\author{
Michael H FREEDMAN \\ DAVID GABAI
}

\begin{abstract}
There exists an open 3-manifold $M$ and a simple closed curve $\gamma \subset M$ such that $\pi_{1}(M \backslash \gamma)$ is infinitely generated, $\pi_{1}(M)$ is finitely generated and the preimage of $\gamma$ in the universal covering of $M$ is equivalent to the standard locally finite set of vertical lines in $\mathbb{R}^{3}$, that is, the trivial link of infinitely many components in $\mathbb{R}^{3}$.
\end{abstract}

57N10; 57M10, 57N45

\section{Introduction}

Definition 0.1 We say that the locally finite collection of proper lines $\Gamma \subset \mathbb{R}^{3}$ is a trivial $\mathbb{R}^{3}$-link if there exists a homeomorphism of $\mathbb{R}^{3}$ taking $\Gamma$ to a subset of $(\mathbb{Z}, 0) \times \mathbb{R} \subset \mathbb{R}^{2} \times \mathbb{R}$.

For example, if $L$ is a locally finite union of geodesics in $\mathbb{H}^{3}$, then $L$ is a $\mathbb{R}^{3}$-trivial link, as seen by applying Morse theory to the distance function from any point in $\mathbb{T}^{3}$.

The main result in this paper is the following:

Theorem 0.2 There exists a simple closed curve $\gamma$ in an open 3-manifold $M$ such that

(1) $\pi_{1}(M-\gamma)$ is infinitely generated,

(2) $\pi_{1}(M)$ is finitely generated,

(3) the universal covering $\widetilde{M}$ of $M$ is $\mathbb{R}^{3}$ and

(4) the preimage $\Gamma$ of $\gamma$ in $\widetilde{M}$ is $\mathbb{R}^{3}$-trivial.

Addendum 0.3 A simple closed curve $\omega$ can be chosen in the above manifold $M$ satisfying the above properties as well as the following additional ones:

(1) $\omega$ is algebraically disc busting in $\pi_{1}(M)$ and

(2) $0=[\omega] \in H_{1}\left(M, \mathbb{Z}_{2}\right)$. 
Definition 0.4 A nontaming knot is a smooth simple closed curve $k$ in a 3-manifold $M$ such that $\pi_{1}(M)$ is finitely generated and $\pi_{1}(M-k)$ is infinitely generated.

Remarks 0.5 By Tucker [8], the condition $\pi_{1}(M-\gamma)$ is infinitely generated implies that the manifold $M$ is not tame, that is, not the interior of a compact manifold. There are lots of examples of nontame manifolds with finitely generated fundamental group whose universal covers are $\mathbb{R}^{3}$, for example, see Theorem 2.1 . This paper provides the first example of a knot in such a manifold, which is sufficiently complicated to be nontaming, yet sufficiently straight to lift to an $\mathbb{R}^{3}$-unlink.

Our manifold $M$ is obtained as a nested union of handlebodies of genus $2, V_{1} \subset V_{2} \subset$ $V_{3} \subset \cdots$ where the inclusion $V_{i} \subset V_{i+1}$ is as in Figure 1. Let $\gamma \subset V_{1}$ be the knot also shown in Figure 1.

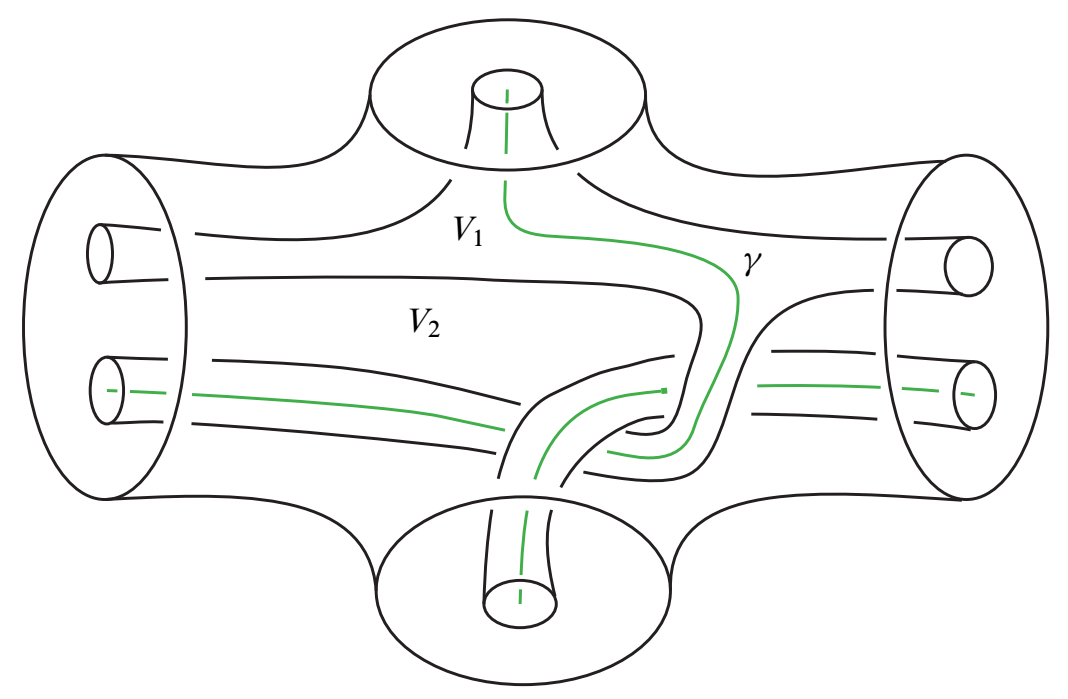

Figure 1: Glue the top disc to the bottom one and the left disc to the right one to obtain the embedding of $V_{1}$ into $V_{2}$.

The paper is organized as follows. In Section 1 we show that $M$ is homotopy equivalent, but not homeomorphic to the standard open genus-2 handlebody and that $\pi_{1}(M-\gamma)$ is infinitely generated. In Section 2 we show that $\Gamma$ is the trivial $\mathbb{R}^{3}$-link of infinitely many components. In Section 3 we prove Addendum 0.3.

Historical Remarks In the early 1990s the first author showed that the nonexistence of a knot having the properties stated in our main result implies the Tame Ends conjecture (also known as the Marden conjecture [4]) for hyperbolic 3-manifolds. See Myers [5]. 
In the fall of 1996 the authors found the knot $\gamma \subset M$. We are finally presenting its proof. Very recently, we found the example of Addendum 0.3.

Ian Agol [1] and independently Danny Calegari and the second author [2] have obtained proofs of the Tame Ends conjecture.

Notation 0.6 If $X \subset Y$, then $N(X)$ denotes a regular neighborhood of $X$ in $Y$. If $X$ is a topological space, then $|X|$ denotes the number of components of $X$.

Acknowledgements The second author was partially supported by NSF grant DMS0071852.

\section{$1 \pi_{1}(M-k)$ is infinitely generated}

Since the inclusion of each $V_{i}$ into $V_{i+1}$ is a homotopy equivalence, it follows that the inclusion of $V_{1}$ into $M$ is a homotopy equivalence and hence $M$ is an open genus-2 homotopy handlebody.

To show that $\pi_{1}(M-k)$ is infinitely generated it suffices to show that $\partial V_{3}$ is incompressible in $V_{3}-\gamma$ and for each $i, \partial\left(V_{i+1}-\stackrel{\circ}{V}_{i}\right)$ is incompressible in $V_{i+1}-\stackrel{\circ}{V}_{i}$ and $V_{i+1}-\stackrel{\circ}{V}_{i}$ is not a product. See Figure 2 . These facts, together with the work of Stallings [7] show that the induced map $\pi_{1}\left(\partial V_{i}\right) \rightarrow \pi_{1}\left(V_{i+1}-\stackrel{\circ}{V}_{i}\right)$ is injective but not surjective. The standard Seifert-Van Kampen argument completes the proof.

Lemma 1.1 $\partial V_{3}$ is incompressible in $V_{3}-\gamma$.

Proof It suffices to show that if $W_{0}=V_{3}-\stackrel{\circ}{N}(\gamma)$, then $R_{0}:=\partial V_{3}$ is incompressible in $W_{0}$. Let $D_{3} \subset V_{3}$ (resp. $E \subset V_{3}$ ) be the disc obtained by gluing $D_{3}^{\prime}$ to $D_{3}^{\prime \prime}$ (resp. $E_{1}$ to $\left.E_{2}\right)$. By considering boundary compressions it suffices to show that if $W_{1}$ is $W_{0}$ split along $D_{3}$ and $R_{1}$ is $R_{0}$ split along $D_{3}$, then $R_{1}$ is incompressible in $W_{1}$. Let $W_{2}$ (resp. $R_{2}$ ) denote $W_{1}$ (resp. $R_{1}$ ) split along $E$. We abuse notation by now viewing $D_{3}^{\prime}, D_{3}^{\prime \prime}$ (resp. $E_{1}, E_{2}$ ) as compact annuli (resp. pants). Note that $R_{2}$ is incompressible in $W_{2}$, for any essential compressing disc $H$ would nontrivially separate the set $\left\{D_{3}^{\prime}, D_{3}^{\prime \prime}, E_{1}, E_{2}\right\}$. On the other hand by considering $\partial N(\gamma) \cap W_{2}$ we see that all of these components must lie in the same component of $W_{2}-H$.

Therefore to show that $R_{1}$ is incompressible in $W_{1}$ it suffices to show that there exists no essential, properly embedded disc $(F, \partial F) \subset\left(W_{2}, E_{1} \cup E_{2} \cup R_{2}\right)$ such that $\partial F \cap\left(E_{1} \cup E_{2}\right)$ is connected. We now show that $F \cap E_{1}=\varnothing$. A similar argument will show that $F \cap E_{2}=\varnothing$. In the natural way write $W_{2}$ as $P \times[1,2]$ where $P$ is a 


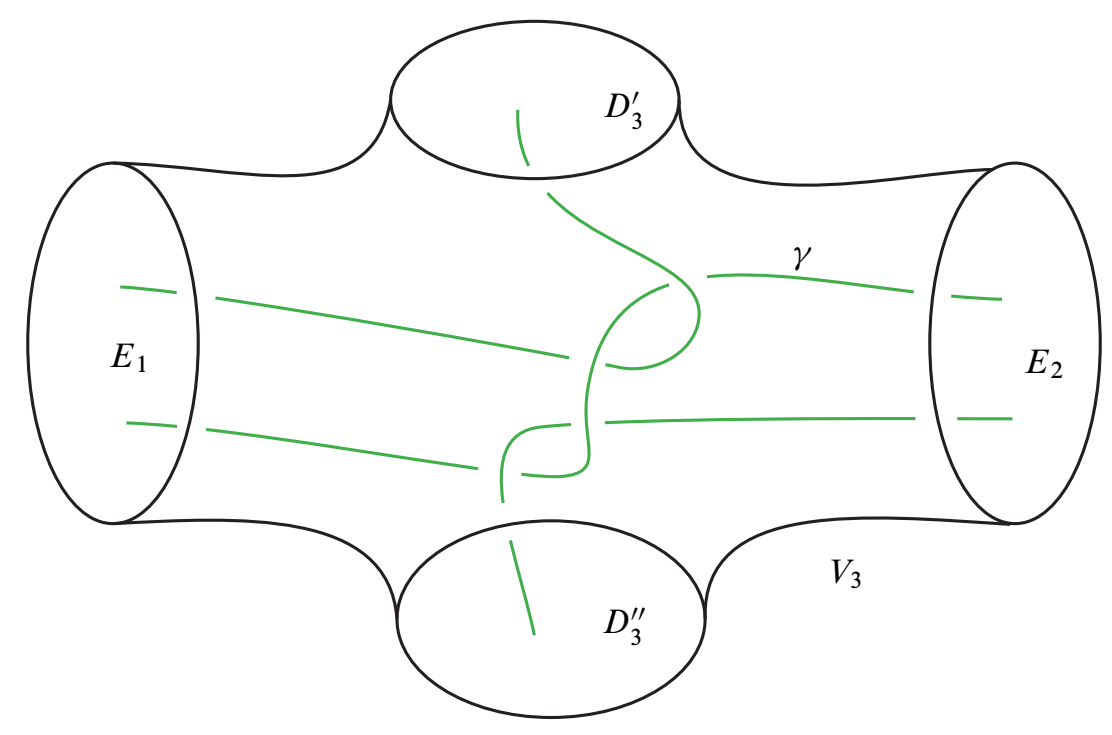

Figure 2: The knot $\gamma$ viewed inside $V_{3}$

disc with 3 open discs removed and $D_{3}^{\prime \prime} \cup E_{1} \subset \stackrel{\circ}{P} \times 1$ and $D_{3}^{\prime} \cup E_{2} \subset \stackrel{\circ}{P} \times 2$. Here $P_{i}$ denotes $P \times i$. Assume that $F$ was chosen so that $\left|F \cap P_{2}\right|$ is minimal and that $F \cap\left(R_{2} \cap \partial P \times[1,2]\right)$ are arcs from $P_{1}$ to $P_{2}$. Isotope $F$ to be Morse with respect to projection onto the [1,2] factor. Arguing as in Roussarie [6] we can assume that the only critical points are of index -1 . Since $F$ is disjoint from $E_{2} \cup D_{3}^{\prime}$ and $F$ is a disc it follows that $F \cap P_{2}$ is a finite union of parallel arcs and the closest saddle point to $P_{2}$ must involve distinct such arcs. Therefore, if $F$ contained a saddle tangency, then by considering a boundary compression we could have found another essential $F$ as above, with $|F \cap P \times 2|$ reduced. It follows that $F$ has no saddle tangencies and hence $\left|F \cap E_{1}\right| \geq 3$, which is a contradiction. See Figure 3 .

Remark 1.2 Another way to prove Lemma 1.1 is to show that the manifold obtained by doubling $V_{3}-\stackrel{\circ}{N}(\gamma)$ along $\partial V_{3}$ is irreducible. One can prove irreducibility of the double by constructing a taut sutured manifold hierarchy.

Note that $\partial V_{2}$ is compressible in $V_{2}-\gamma$.

Since the inclusion of $V_{i}$ into $V_{i+1}$ is a homotopy equivalence we obtain the following:

Lemma 1.3 For each $i \geq 1, V_{i+i}-\stackrel{\circ}{V}_{i}$ has incompressible boundary. 


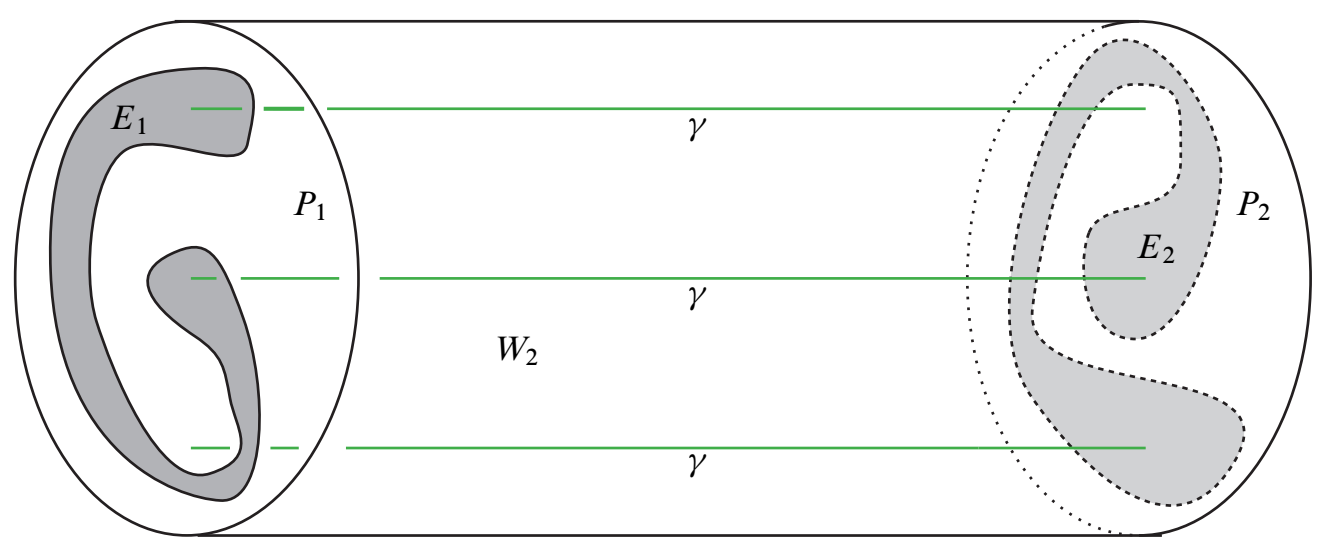

Figure 3

Lemma 1.4 For $i \geq 1, V_{i+1}-\stackrel{\circ}{V}_{i}$ is not a product.

Proof It suffices to consider the case $i=2$. If $V_{3}-\stackrel{\circ}{V}_{2}$ is a product, then the pair $\left(V_{3}, \gamma\right)$ is homeomorphic to $\left(V_{2}, \gamma\right)$. On the other hand, the note after Remark 1.2 implies that $\partial V_{2}$ is compressible in $V_{2}-\gamma$, while Lemma 1.1 implies that $\partial V_{3}$ is incompressible in $V_{3}-\gamma$.

Here is a second proof. Let $W=V_{3}-\stackrel{\circ}{V}_{2}$. Let $(W, \sigma)$ be the sutured manifold with $R_{-}(\sigma)=\partial V_{2}$ and $R_{+}(\sigma)=\partial V_{3}$. It suffices to construct a taut sutured manifold hierarchy $(W, \sigma)=\left(N_{0}, \sigma_{0}\right) \rightarrow\left(N_{1}, \sigma_{1}\right) \rightarrow \cdots \rightarrow\left(D^{2} \times I, \partial D^{2} \times I\right)$ such that for some $j, R_{+}\left(\sigma_{j}\right)$ is not homeomorphic to $R_{-}\left(\sigma_{j}\right)$, since by [3] a taut sutured manifold decomposition of a product always yields a product. (Products are exactly those taut sutured manifolds of minimal complexity, and taut splittings do not increase complexity.) Figure 4 shows a step in such a hierarchy. The top sutured manifold $\left(N_{2}, \sigma_{2}\right)$ is $(W, \sigma)$ split along the product annulus $D_{3}-V_{2}$ followed by splitting along a product disc (that is, a disc crossing the sutures twice) meeting $E_{2}-V_{2}$ in a single arc. The thick brown lines denote the sutures. Note that each of $R_{+}\left(\sigma_{2}\right), R_{-}\left(\sigma_{2}\right)$ is a pant. To obtain $\left(N_{3}, \sigma_{3}\right)$ split along the annulus corresponding to $A_{2}$ and $A_{1}$, so that $A_{2}$ is given the +- orientation. Note that $R_{+}\left(\sigma_{3}\right)$ is not homeomorphic to $R_{-}\left(\sigma_{3}\right)$. Splitting $\left(N_{3}, \sigma_{3}\right)$ along a product disc yields $\left(N_{4}, \sigma_{4}\right)$ where $N_{4}=D^{2} \times S^{1}$ and the sutures of $\sigma_{4}$ are 4 parallel longitudes. One more splitting yields, $\left(D^{2} \times I, \partial D^{2} \times I\right)$. 


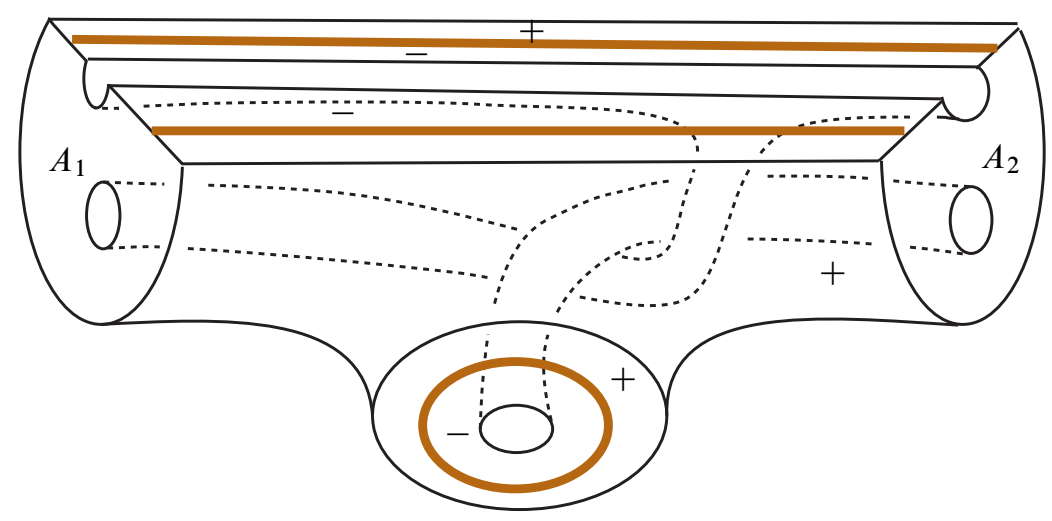

Glue annuli $A_{1}$ to $A_{2}$ to obtain $\left(N_{2}, \sigma_{2}\right)$.

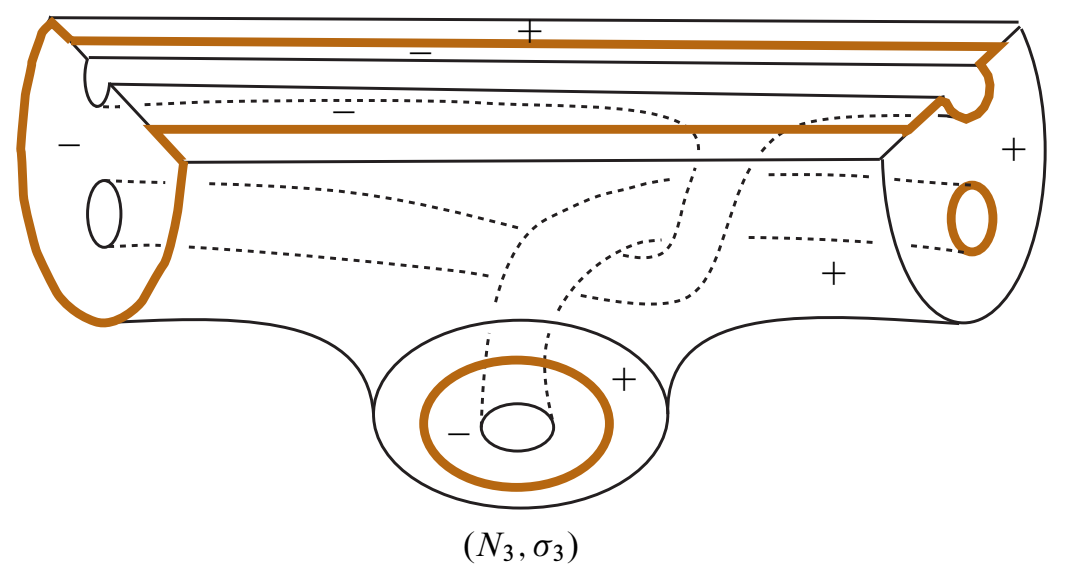

Figure 4

\section{$2 \Gamma$ is $\mathbb{R}^{3}$-trivial}

The following is well known.

Theorem 2.1 If the open 3-manifold $N$ is exhausted by compact irreducible manifolds $W_{1} \subset W_{2} \subset \cdots$ such that for each $i, \operatorname{in}_{*}: \pi_{1}\left(W_{i}\right) \rightarrow \pi_{1}\left(W_{i+1}\right)$ is injective, then $\tilde{N}=\mathbb{R}^{3}$. 
Proof The universal covering space $\tilde{N}$ of $N$ is exhausted by the universal covering spaces of the various $W_{i}$ 's. By Waldhausen [9], the universal covering space of $W_{i}$ is $B^{3}-K_{i}$, where $K_{i} \subset \partial B^{3}$ is compact. Since a space is $\mathbb{R}^{3}$ if every compact set lies in a 3 -cell, the result follows.

Lemma 2.2 If $L$ is a smooth locally finite link in the open unit 3-ball $B \subset \mathbb{R}^{3}$, such that away from exactly one point, each component is transverse to the concentric 2-spheres, then $L$ is $\mathbb{R}^{3}$-trivial.

Corollary 2.3 A locally finite collection of geodesics in $\mathbb{\boxplus}^{3}$ is $\mathbb{R}^{3}$-trivial.

Definition 2.4 Let $T$ denote the standard infinite $\mathbb{R}^{3}$-link $(\mathbb{Z}, 0) \times \mathbb{R} \subset \mathbb{R}^{2} \times \mathbb{R}$. Let $X$ be the 3 -manifold with boundary obtained by removing small open regular neighborhoods of the rays $\mathcal{R}_{X}:=(\mathbb{Z}, 0) \times[1, \infty) \cup(\infty,-1]$. Let $T_{X}$ be the restriction of $T$ to $X$.

Remark 2.5 If $X_{1}$ is the 3-manifold with boundary obtained from the standard infinite $\mathbb{R}^{3}$-link by removing small open regular neighborhoods of the rays $\mathcal{R}_{X_{1}}$ defined by $\{(n, 0) \times[n, \infty) \cup(-\infty, n-1] \mid n \in \mathbb{Z}\}$ and $T_{X_{1}}$ is the restriction of $T$ to $X_{1}$, then $\left(X_{1}, T_{X_{1}}\right)$ is diffeomorphic to $\left(X, T_{X}\right)$.

The pair $\left(X, T_{X}\right)$ can be viewed geometrically via the following lemma.

Lemma 2.6 Let $G$ be the 2-dimensional Schottky group generated by length 10 translations $g_{1}, g_{2}$ along orthogonal geodesics $A, B \subset \mathbb{U}^{2}$. Extend $G$ to act on $\mathbb{M}^{3}$. Let $Q \subset \mathbb{M}^{3}$ be the totally geodesic plane orthogonal to $B$ at distance 5 from $A \cap B$ and $\mathcal{Q}$ be the orbit $G Q$. Let $Y$ be the closure of a component of $\mathbb{M}^{3}-\mathcal{Q}$ and $G B_{Y}$ the restriction of the orbit $G B$ to $Y$. Then there is a diffeomorphism $\left(X, T_{X}\right) \rightarrow\left(Y, G B_{Y}\right)$.

Let $\pi: \widetilde{M} \rightarrow M$ denote the universal covering projection and let $\Gamma$ denote the link $\pi^{-1}(\gamma)$. By Theorem $2.1 \widetilde{M}$ is homeomorphic to $\mathbb{R}^{3}$.

The construction of $M$ gives rise to a properly embedded plane $P \subset M$ which intersects each $V_{i}$ in a single disc $D_{i}$ and intersects $V_{3}$ in the disc $D_{3}$. Furthermore $P \cap \gamma=D_{3} \cap \gamma$. Let $\mathcal{P}=\pi^{-1}(P)$.

Proposition 2.7 There exists a diffeomorphism $(\widetilde{M}, \Gamma) \rightarrow\left(\mathbb{M}^{3}, G B\right)$. 
Assuming for the moment Proposition 2.7 we obtain the following proof:

Proof that $\Gamma$ is $\mathbb{R}^{3}$-trivial It follows from the Proposition 2.7 that the pair $(\widetilde{M}, \Gamma)$ is diffeomorphic to $\left(\mathbb{T}^{3}, \Delta\right)$ where $\Delta$ is a locally finite union of pairwise disjoint geodesics. By Lemma 2.2, $\Gamma$ is $\mathbb{R}^{3}$-trivial.

Proof of Proposition 2.7 It suffices to show that if $W$ is the closure of a component of $\widetilde{M}-\mathcal{P}$ and $\Gamma_{W}$ is the restriction of $\Gamma$ to $W$, then there is a diffeomorphism $\left(W, \Gamma_{W}\right) \rightarrow\left(Y, G B_{Y}\right)$ where $Y$ and $G B_{Y}$ are as in Lemma 2.6. By Lemma 2.6 it suffices to show that $\left(W, \Gamma_{W}\right)$ is diffeomorphic to $\left(X, T_{X}\right)$, where $T_{X}$ is defined as in Definition 2.4. Let $W_{i}$ denote the compact manifold obtained by splitting $V_{i}$ open along $D_{i}$. Then $W$ is exhausted by the manifolds $\tilde{V}_{i}$.

Consider the $\mathbb{R}^{3}$-link $\Sigma$ shown in Figure 5 . It has infinitely many components and is invariant under a rigid $\mathbb{R}^{3}$-translation $g$. Each component has an end which is a vertical ray and another that forever spirals down. Let $\mathcal{R}$ be the union of the (thick) blue rays, two for each component of $\Sigma$. Let $N(\mathcal{R})$ be a union of small open regular neighborhoods of the components of $\mathcal{R}$ and $Z=\mathbb{R}^{3}-\stackrel{\circ}{N}(\mathcal{R})$. The pair $\left(W, \Gamma_{W}\right)$ is diffeomorphic to $(Z, \Sigma \cap Z)$. Indeed, $Z$ can be exhausted by manifolds diffeomorphic to $\tilde{V}_{i}$ in a manner compatible with the inclusion $\tilde{V}_{i} \subset \tilde{V}_{i+1}$. Furthermore, the quotient $Z /\langle g\rangle=W / \mathbb{Z}$ where $\mathbb{Z}$ is the group of covering translations of $W$ and $\tilde{V}_{i} / \mathbb{Z}=V_{i}$. Figure 6 shows three fundamental domains $V_{1}$ within $Z$. Figure 7 shows one fundamental domain of $V_{2}$. Notice that the curves $\alpha$ and $\beta$ bound discs in the boundary of this fundamental domain which lie in $\partial Z$. Again, just translate by $g$ to get the entire embedding of $\tilde{V}_{2} \subset Z$. In a similar manner construct $\tilde{V}_{i}, i \geq 3$.

Consider the collection $\left\{H_{i}\right\}, i \in \mathbb{Z}$ of horizontal planes shown as lines in Figure 8. Coordinates on $\mathbb{R}^{3}$ could have been chosen so that $H_{i}=\mathbb{R}^{2} \times i$ and $g\left(H_{i}\right)=H_{i+1}$. If $S_{i}$ is the slab $\mathbb{R}^{2} \times[i, i+1]$, then $\Sigma \mid S_{i}$ is equivalent to the link $(\mathbb{Z}, 0) \times[i, i+1]$. Putting these slabs together, we conclude that $\Sigma$ is $\mathbb{R}^{3}$-trivial.

The diffeomorphism $H$ of $\mathbb{R}^{3}$ which takes $\Sigma$ to the standard link $T$ could have been chosen to fix $H_{0}$ pointwise and setwise fix the various horizontal planes. Therefore it could have been chosen to take $\mathcal{R}$ to the rays $\mathcal{R}_{X_{1}}$. This shows that $\left(W, \Gamma_{W}\right)$ is diffeomorphic to $\left(X_{1}, T_{X_{1}}\right)$ and hence is diffeomorphic to $\left(X, T_{X}\right)$.

\section{Another example}

Theorem 0.2 answers in the negative a conjecture of the first author. Myers [5] asked whether a more restrictive version of that conjecture holds. The example of this section 


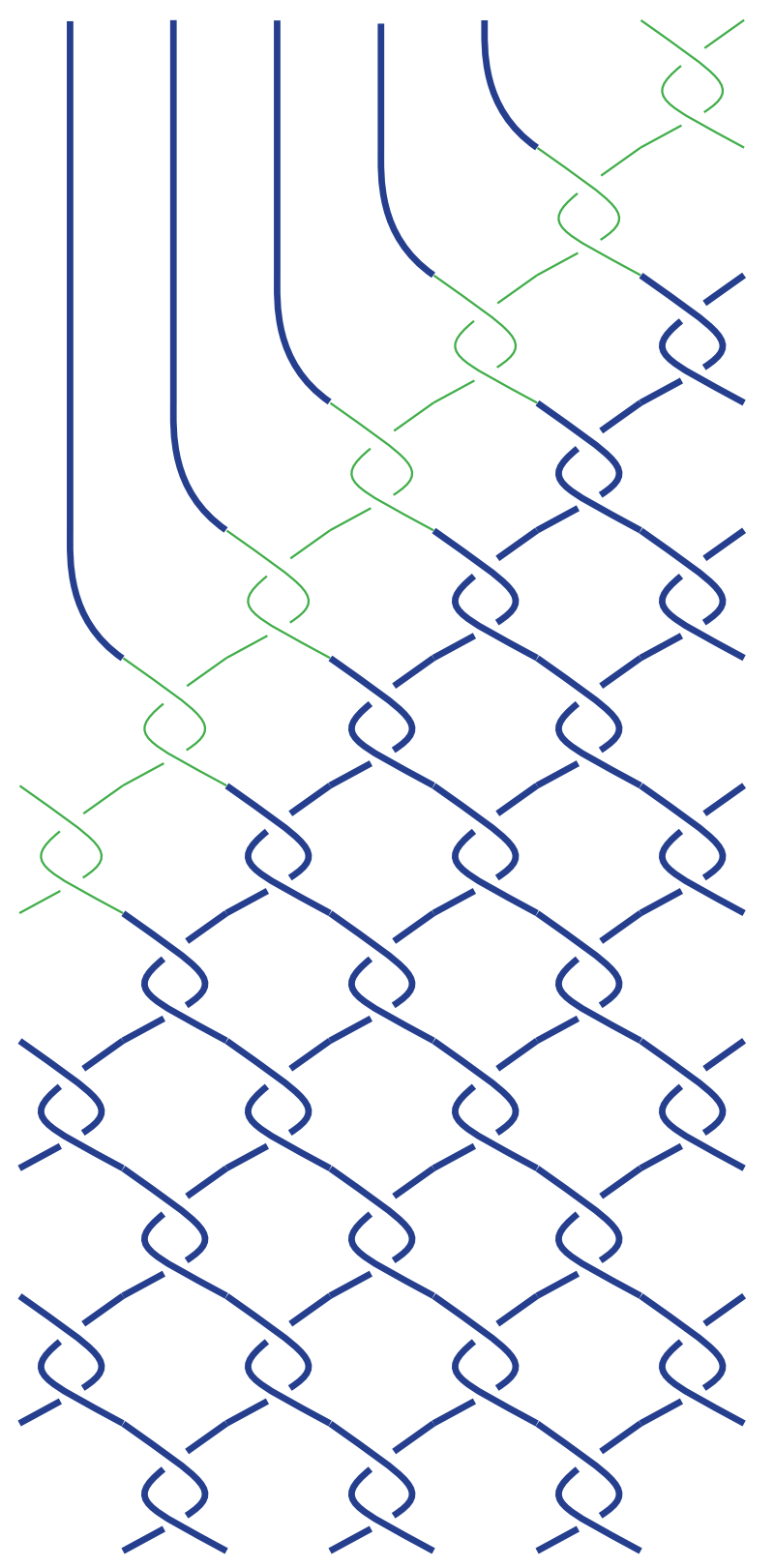

Figure 5: This infinite $\mathbb{R}^{3}$-link is rigidly $\mathbb{Z}$-translation invariant. The top part of each strand is straight and the bottom part is infinitely twisted in the helical pattern indicated. 


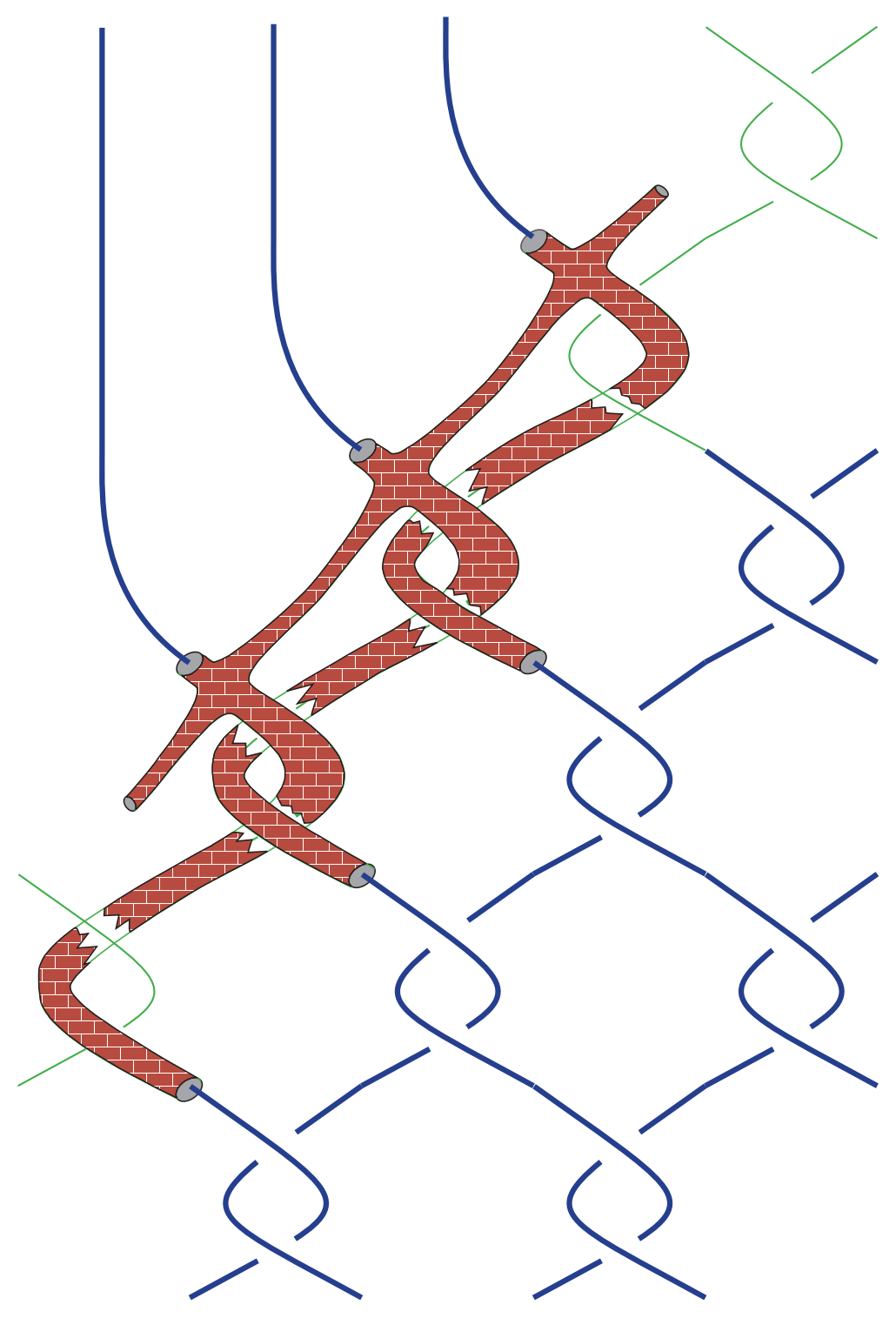

Figure 6: Three fundamental domains of $V_{1}$ lifted to $Z$

provides a similar answer to that question. Let the manifold $M$ be as in Section 2, with the knot $\omega \subset V_{1}$ presented as in Figure 9.

Proof of Addendum 0.3 With respect to the standard generators of $\pi_{1}\left(V_{1}\right)$, $\omega$ represents the element $a^{2} b^{2}$ which according to Myers [5] is algebraically disc busting in 


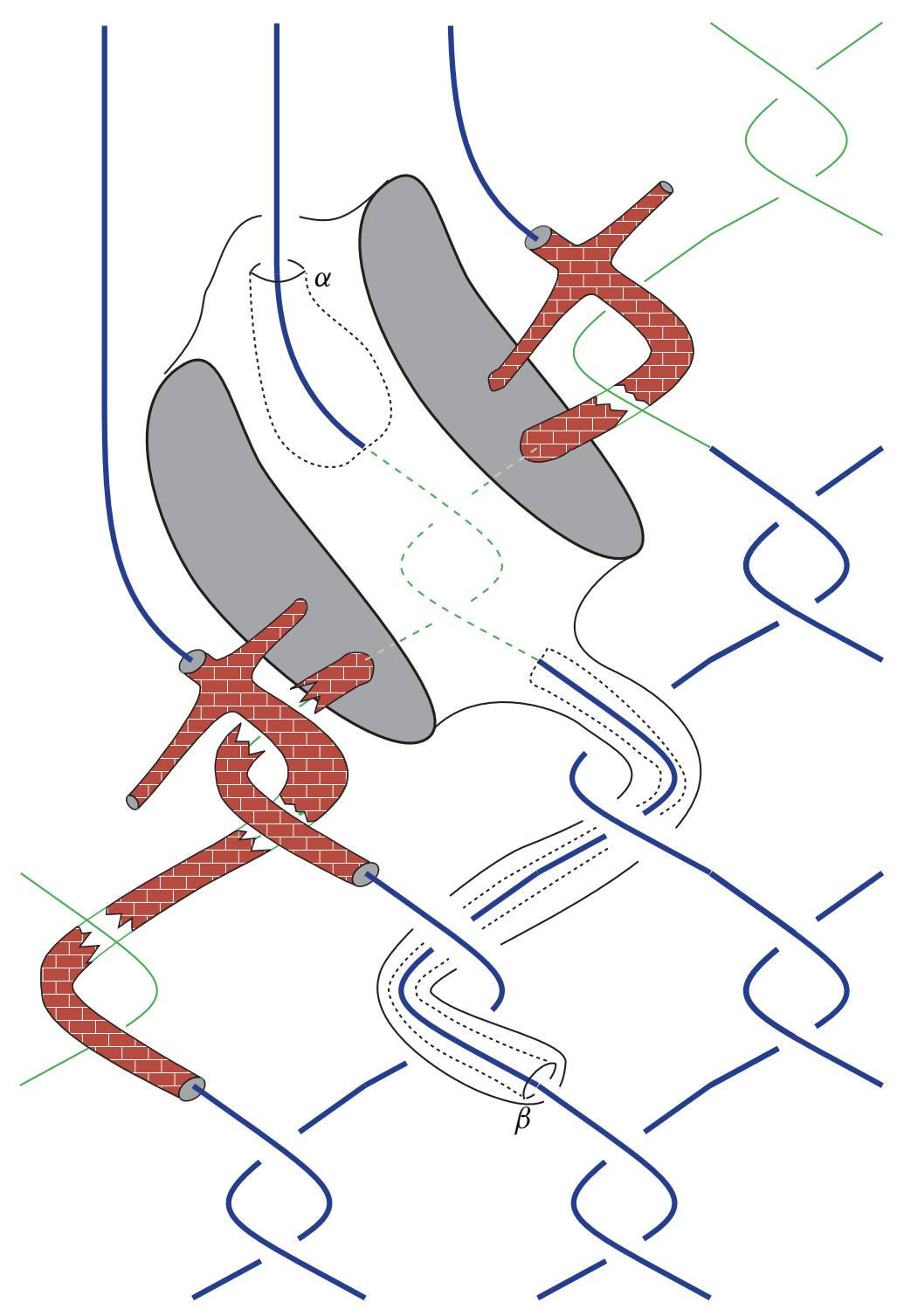

Figure 7: One fundamental domain of $\widetilde{V}_{2}$ lifted to $Z$

$V_{1}$ and hence in $M$. that is, $\pi_{1}(M)$ cannot be expressed as a nontrivial free product such that $[\omega]$ can be conjugated to lie in a single factor. Since $\omega$ is algebraically disc busting, $\partial V_{1}$ is incompressible in $V_{1}-\omega$. As in Section $1, \pi_{1}(M-\omega)$ being infinitely generated then follows from Lemma 1.3 and Lemma 1.4.

Algebraic ${ }^{3} \mathcal{G}$ Geometric Topology, Volume 7 (2007) 


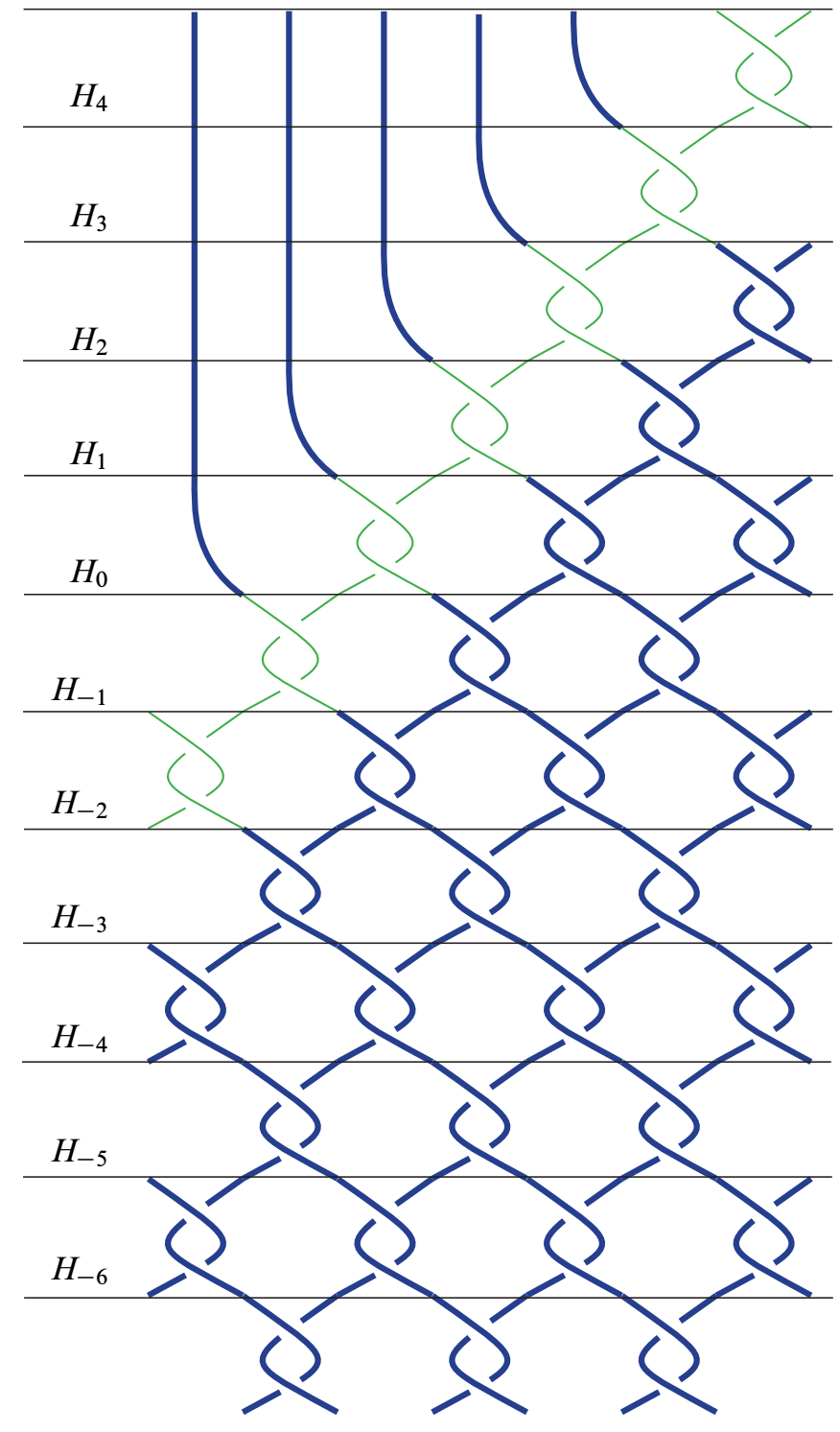

Figure 8

An argument similar to that of Section 2 shows that the restriction of $\Omega$ to $W$ is the union of properly embedded arcs $\Omega_{W}$ as drawn in Figure 10. Figure 10 can be decoded with the help of Figure 11, for example to unclutter the picture, certain pairs of thin green arcs are drawn as one arc. Note that $W$ is $\mathbb{R}^{3}$ with open regular neighborhoods 


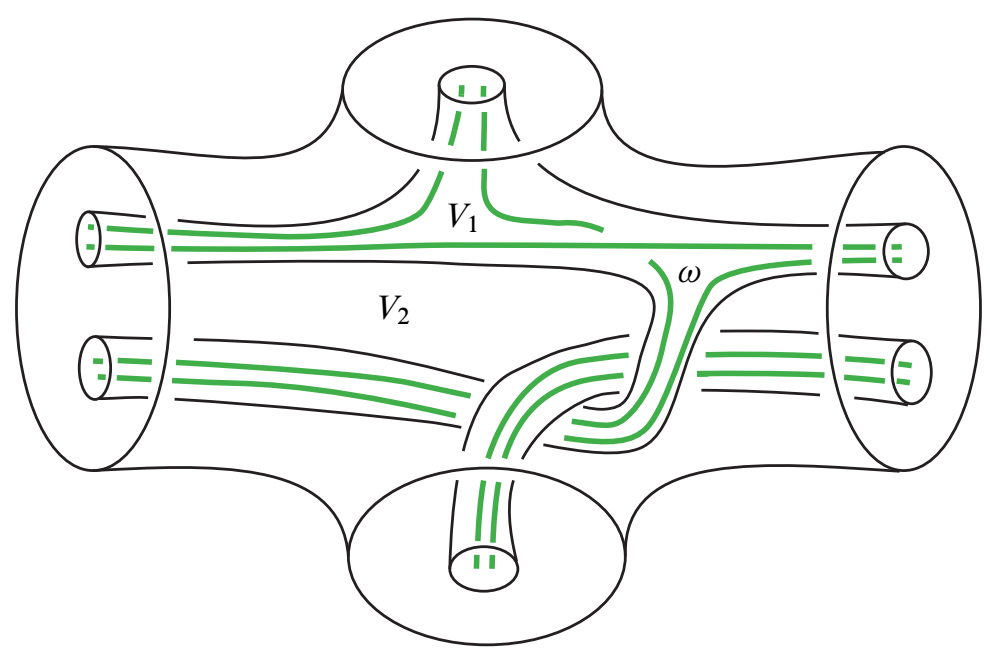

Figure 9

of a countable collection of rays deleted. These neighborhoods are denoted by the thick blue lines. Finally the boxes drawn in Figure 10 coordinatize $\mathbb{R}^{3}$ and will be useful for the next paragraph. Imagine that both $\partial W$ and $\Omega_{W}$ lie very close to the $x y$-plane. Let $\left\{E_{i}\right\}$ denote the components of $\partial W$.

To each component $E$ of $\partial W$ we define a foliation $\mathcal{F}_{E}$ of $W$ which is the restriction of a topologically concentric foliation on $\mathbb{R}^{3}$ with center in the component of $\mathbb{R}^{3}-W$ separated off by $E$. For each $i, \mathcal{F}_{E} \mid E_{i}$ will be a topologically concentric foliation by circles with center point the dot shown in Figure $12 . \mathcal{F}_{E}$ will have exactly one tangency with each component of $\Omega_{W}$ except for the two components $\Omega_{E}$ which hit $E$ and $\mathcal{F}_{E}$ will be transverse to $\Omega_{E}$. Suppose that $E$ is the component containing the point $(0,0)$ shown in Figure 10. The leaves $S_{t}$ of $\mathcal{F}_{E}$ will be parametrized by $[0, \infty)$, where $S_{0}$ is a point. Define $S_{i}, i \in \mathbb{N}$ according to the pattern given in Figure 12 . Next modify these spheres as in Figure 13. In particular if $S_{i} \cap E_{j} \neq \varnothing$, then $S_{i} \cap E_{j}$ is a circle. The other spheres get modified in a similar way. For example, the modified $S_{3}$ has three tube like extensions. One passes by $(1,1)$ and the others at $(2,2)$ and $(3,3)$. It is an exercise to show that the desired foliation $\mathcal{F}_{E}$ can be constructed to contain these integral spheres. Note that near $(0,0)$, but not including $(0,0)$, all the leaves of $\mathcal{F}_{E}$ are discs.

In a similar way construct a foliation $\mathcal{F}_{0}$ on $W$ to have all the properties of $\mathcal{F}_{E}$ except that the center point of the concentric foliation lies in $\operatorname{int}\left(W-\Omega_{W}\right)$, nearby leaves are spheres and each component of $\Omega_{W}$ is tangent to $\mathcal{F}_{0}$ at exactly one point. 


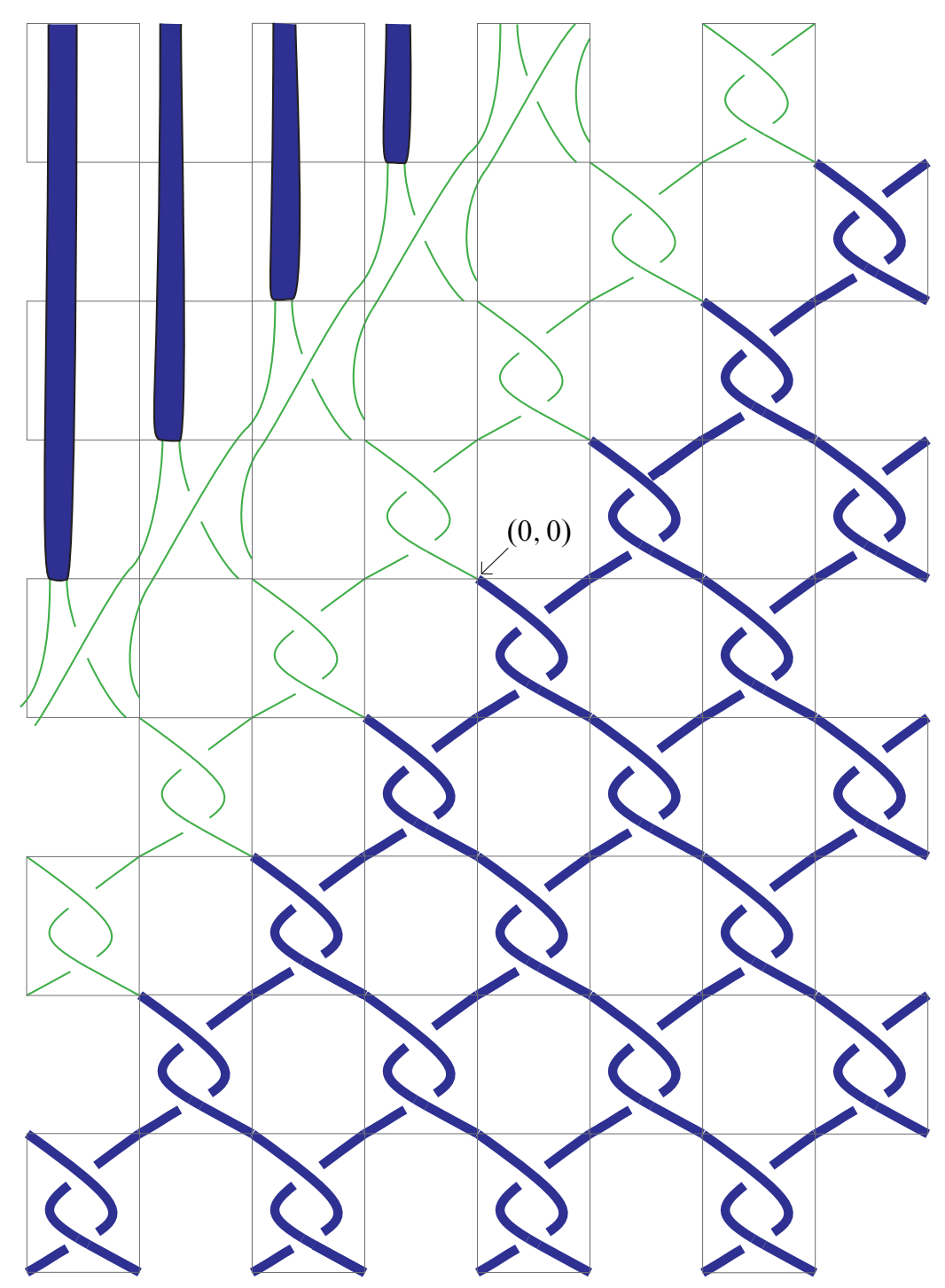

Figure 10

To show that $\Omega$ is $\mathbb{R}^{3}$-trivial we describe a foliation $\mathcal{F}$ on $\widetilde{M}=\mathbb{R}^{3}$ which satisfies the hypothesis of Lemma 2.2 with respect to the link $\Omega$. $\widetilde{M}$ is built by gluing copies of $W$ in a treelike fashion. Let $T$ be the tree dual to $\mathcal{P} \subset \widetilde{M}$ with base vertex $v_{0}$. Let $v_{0}$ also denote the corresponding copy of $W$. Define $\mathcal{F} \mid v_{0}=\mathcal{F}_{0}$. If $v_{i}$ and $v_{0}$ have an edge in common and $v_{i}$ is glued to $v_{0}$ along the plane $E_{g(i)} \subset \partial v_{i}$, then give $v_{i}$ the foliation 


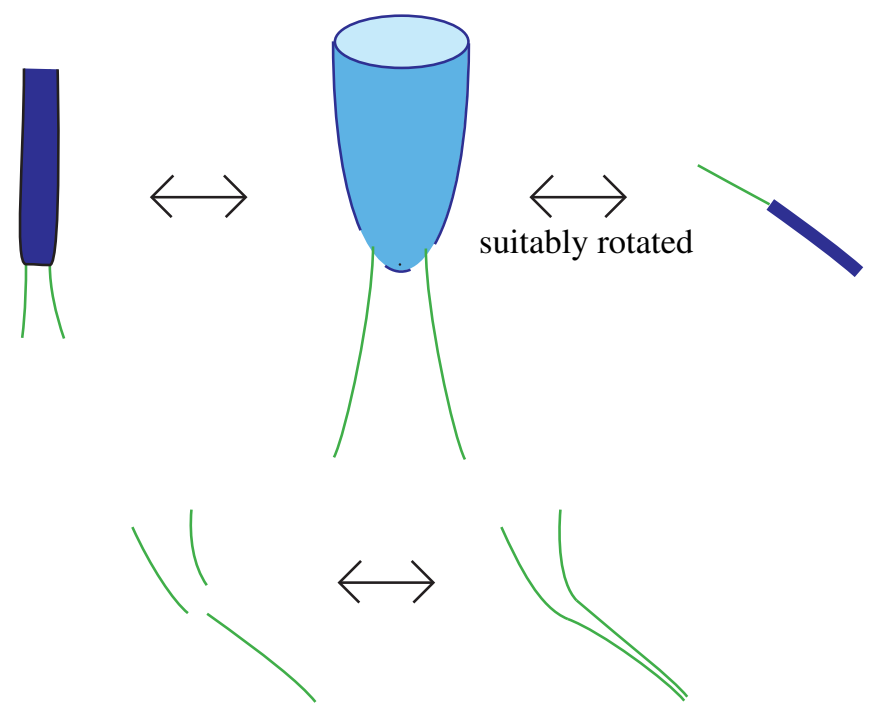

Figure 11

$\mathcal{F}_{E_{g(i)}}$. In what follows we denote $\mathcal{F}_{E_{g(i)}}$ by $\mathcal{F}_{g(i)}$. Since each foliation restricts to a concentric foliation on $E_{g(i)}$ the identification of $\mathcal{F}_{0}$ and $\mathcal{F}_{g(i)}$ is determined by a homeomorphism $h_{0 i}:[0, \infty) \rightarrow[0, \infty)$. Similarly if $v_{j}$ and $v_{k}$ share an edge with $v_{j}$ closer to $v_{0}$, then give $v_{k}$ the foliation $\mathcal{F}_{g(k)}$ where the plane $E_{g(k)} \subset \partial v_{k}$ glues to $v_{j}$. So $\mathcal{F}$ is determined by the various homeomorphisms $h_{i j}:[0, \infty) \rightarrow[0, \infty)$ where $v_{i}$ and $v_{j}$ share an edge. Any choice of functions gives rise to a foliation by spheres and planar surfaces of possibly infinite Euler characteristic. Furthermore, since each leaf of $\mathcal{F}_{E_{i}}$ is compact and hits $E_{i}$ in exactly one component, it follows that each leaf of $\mathcal{F}$ hits $v_{0}$ in exactly one component. If the leaves of $\mathcal{F}_{0}$ are parametrized by $[0, \infty)$ and $T_{0} \subset T_{1} \subset \cdots$ is an exhaustion of $T$ by compact connected sets, then pass to a subsequence of the $T_{i}$ 's and choose the functions $h_{i j}$ so that if $t \leq n-1 \in \mathbb{N}$ and $L_{t}$ is the leaf of $\mathcal{F}$ passing through the leaf of $\mathcal{F}_{0}$ parametrized by $t \in[0, \infty)$, then $L_{t}$ is a sphere contained in $\widetilde{M}_{n}$, where $\widetilde{M}_{n}$ is the submanifold of $\widetilde{M}$ corresponding to $T_{n}$. Assume that $\mathcal{F}$ has been inductively constructed on $\widetilde{M}_{n-1}$ and satisfies the above conditions for $t \leq n-2$. Let $\mathcal{G}$ denote those leaves of $\mathcal{F} \mid \widetilde{M}_{n-1}$ which restrict to leaves $L_{t} \subset \mathcal{F}_{0}$ with $t \in[0, n-1]$. There is a finite set $F=\left\{F_{1}, \cdots, F_{k}\right\}$ of components of $\partial \widetilde{M}_{n-1}$ so that $\mathcal{G} \cap \partial \widetilde{M}_{n-1} \subset F$ and lies in a compact subset $C$ of $F$. By passing to a subsequence we can suppose that each $F_{i}$ glues to a vertex of $T_{n}$. If $v_{j}$ glues to $v_{i} \in T_{n-1}$ along $F_{p}$, where $1 \leq p \leq k$, then choose the function $h_{i j}$ so that each circle $c$ of $\mathcal{G} \mid F_{p}$ is capped off by a disc of $\mathcal{F}_{g(j)}$.

Algebraic 83 Geometric Topology, Volume 7 (2007) 


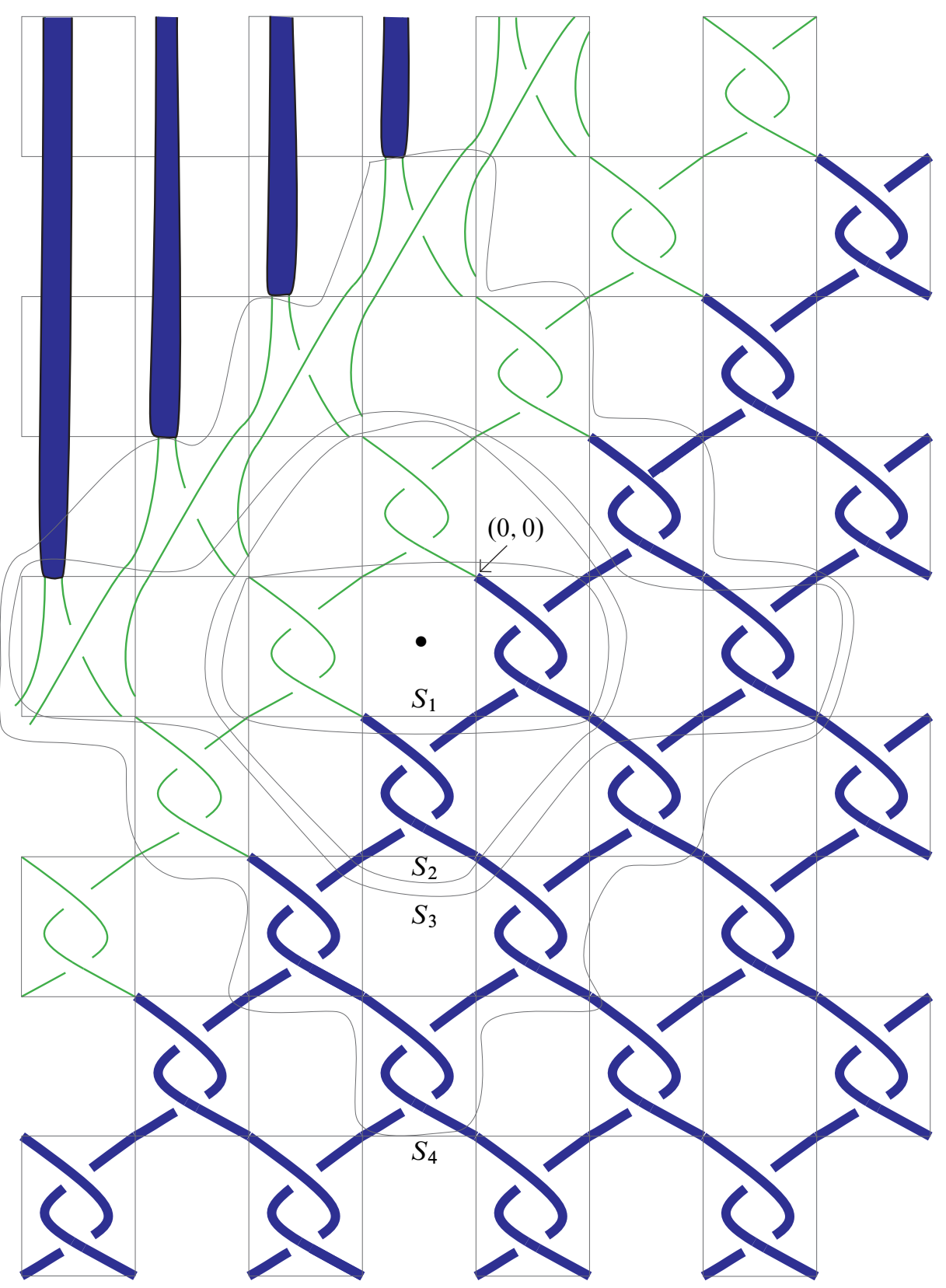

Figure 12

Algebraic $\mathcal{G}^{\mathcal{G}} \mathcal{G}$ eometric $\mathcal{T}$ opology, Volume 7 (2007) 


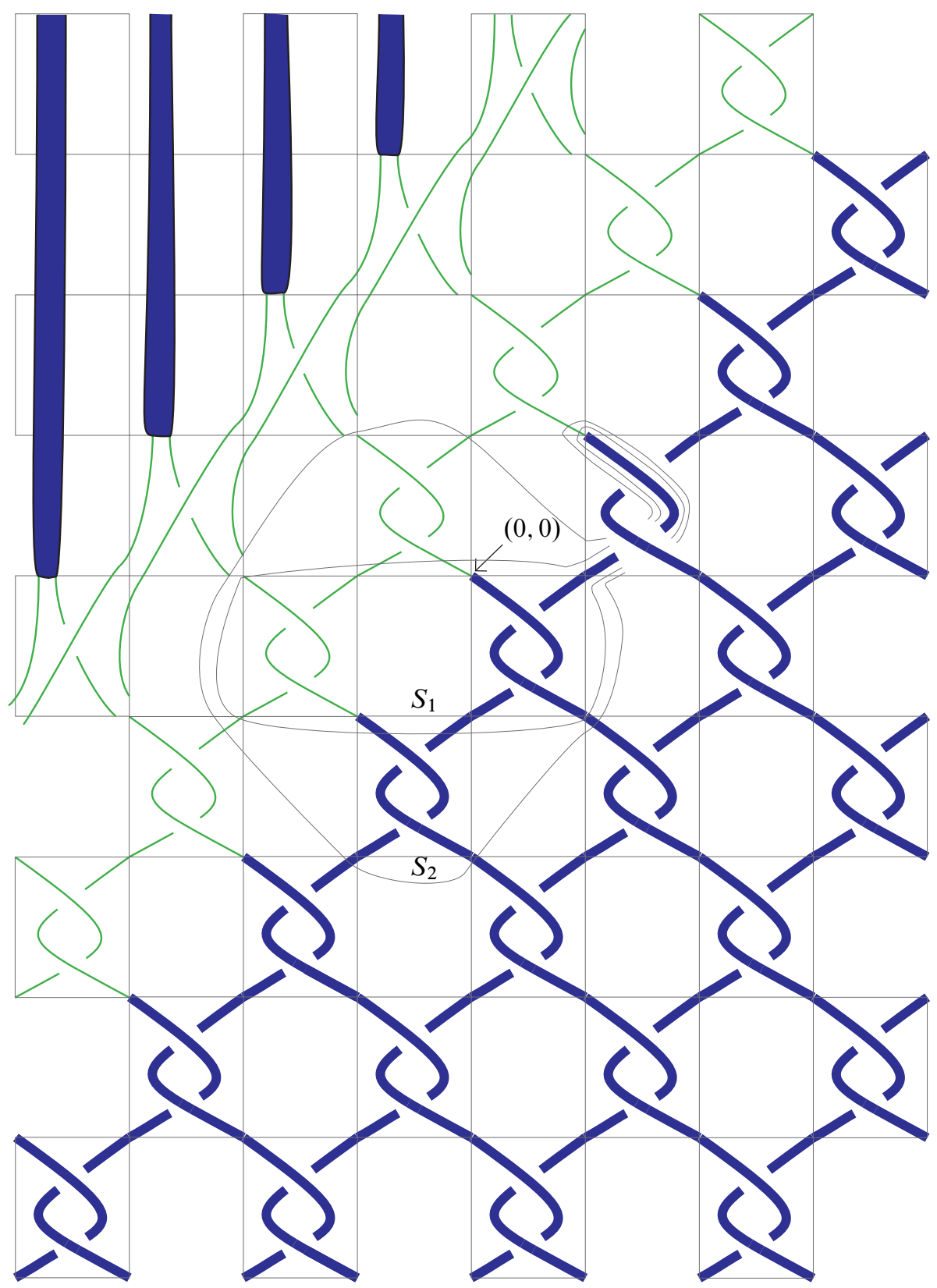

Figure 13

Algebraic $8 \mathcal{G}$ Geometric Topology, Volume 7 (2007) 


\section{References}

[1] I Agol, Tameness of hyperbolic 3-manifolds arXiv:GT/0405568

[2] D Calegari, D Gabai, Shrinkwrapping and the taming of hyperbolic 3-manifolds, J. Amer. Math. Soc. 19 (2006) 385-446 MR2188131

[3] D Gabai, Foliations and the topology of 3-manifolds, J. Differential Geom. 18 (1983) 445-503 MR723813

[4] A Marden, The geometry of finitely generated kleinian groups, Ann. of Math. (2) 99 (1974) 383-462 MR0349992

[5] R Myers, End reductions, fundamental groups, and covering spaces of irreducible open 3-manifolds, Geom. Topol. 9 (2005) 971-990 MR2140996

[6] R Roussarie, Plongements dans les variétés feuilletées et classification de feuilletages sans holonomie, Inst. Hautes Études Sci. Publ. Math. (1974) 101-141 MR0358809

[7] J Stallings, On fibering certain 3-manifolds, from: "Topology of 3-manifolds and related topics (Proc. The Univ. of Georgia Institute, 1961)”, (M Fort, editor), PrenticeHall, Englewood Cliffs, N.J. (1962) 95-100 MR0158375

[8] T W Tucker, Non-compact 3-manifolds and the missing-boundary problem, Topology 13 (1974) 267-273 MR0353317

[9] F Waldhausen, On irreducible 3-manifolds which are sufficiently large, Ann. of Math. (2) 87 (1968) 56-88 MR0224099

Microsoft Corporation

Department of Mathematics, Princeton University

Princeton, NJ 08544

michaelf@microsoft.com, gabai@princeton.edu

Received: 28 May 2005

Algebraic $\&$ Geometric Topology, Volume 7 (2007) 